University of Wollongong

Research Online

Faculty of Engineering and Information

Faculty of Engineering and Information

Sciences - Papers: Part B

Sciences

2018

Thermoelectric and thermal transport properties of graphene under strong magnetic field

Wenye Duan

University of Wollongong, wduan@uow.edu.au

Junfeng Liu

Peking University

C Zhang

University of Wollongong, czhang@uow.edu.au

Zhongshui Ma

Peking University, zma@uow.edu.au

Follow this and additional works at: https://ro.uow.edu.au/eispapers1

Part of the Engineering Commons, and the Science and Technology Studies Commons

Research Online is the open access institutional repository for the University of Wollongong. For further information contact the UOW Library: research-pubs@uow.edu.au 


\title{
Thermoelectric and thermal transport properties of graphene under strong magnetic field
}

\author{
Abstract \\ We investigate theoretically the thermoelectric and thermal transport properties of graphene under strong \\ magnetic field in the presence of short-range scatterers. The numerical results are in good agreement \\ with available experimental data for all thermoelectric quantities except for the Seebeck coefficient near \\ zero chemical potential. This anomaly is attributed to the overestimation of the longitudinal resistivity. \\ Furthermore, we find an anomalous oscillation in the transverse thermal conductivity at the lowest \\ Landau level and double peaks in the longitudinal thermal conductivity at higher Landau levels, which are \\ expected to be observed in future experiments on high mobility graphene samples. An important finding \\ of our work is that the thermoelectric figure of merit $Z T$ can be as high as 2.4 for a magnetic field under \\ which the chemical potential is pinned to the $[n=1]$ Landau level. This finding can greatly advance the \\ thermoelectric application of graphene.

\section{Disciplines} \\ Engineering | Science and Technology Studies

\section{Publication Details} \\ Duan, W., Liu, J., Zhang, C. \& Ma, Z. (2018). Thermoelectric and thermal transport properties of graphene \\ under strong magnetic field. Physica E: Low-Dimensional Systems and Nanostructures, 104 173-176.
}




\title{
Thermoelectric and thermal transport properties of graphene under strong magnetic field
}

\author{
Wenye Duan ${ }^{\mathrm{a}, \mathrm{b}, *}$, Jun-Feng Liuc ${ }^{\mathrm{c}}$, Chao Zhang ${ }^{\mathrm{b}}$, Zhongshui Ma ${ }^{\mathrm{a}, \mathrm{d}}$ \\ ${ }^{a}$ School of Physics, Peking University, Beijing 100871, China \\ ${ }^{b}$ School of Physics, University of Wollongong, New South Wales 2522, Australia \\ ${ }^{c}$ Department of Physics, Guangzhou University, Guangzhou 510405, China \\ ${ }^{d}$ Collaborative Innovation Center of Quantum Matter, Beijing 100871, China
}

\begin{abstract}
We investigate theoretically the thermoelectric and thermal transport properties of graphene under strong magnetic field in the presence of short-range scatterers. The numerical results are in good agreement with available experimental data for all thermoelectric quantities except for the Seebeck coefficient near zero chemical potential. This anomaly is attributed to the overestimation of the longitudinal resistivity. Furthermore, we find an anomalous oscillation in the transverse thermal conductivity at the lowest Landau level and double peaks in the longitudinal thermal conductivity at higher Landau levels, which are expected to be observed in future experiments on high mobility graphene samples. An important finding of our work is that the thermoelectric figure of merit $Z T$ can be as high as 2.4 for a magnetic field under which the chemical potential is pinned to the $|n|=1$ Landau level. This finding can greatly advance the thermoelectric application of graphene.
\end{abstract}

Keywords: graphene, thermoelectric transport, thermal transport

PACS: 72.80.Vp, 72.15.Jf, 73.50.Lw

\section{Introduction}

Since the experimental discovery of graphene in 2004 [1], researching its novel properties has reveled many potential applications in electronics and photonics, but in a less extent in its thermoelectrics properties. The electrical and thermal transport properties of graphene can be influenced strongly by the external conditions, such as the bias, sample geometry, and the impurity scatterings[2-5]. Especially, the electrical transport under magnetic field shows a large amount of novel features, such as the half integer quantum Hall effect[2, 6], metal to insulator transition on the zeroth Landau level (LL) [7] correlated with additional quantum Hall states due to the split of valley and spin degeneracies above 10T magnetic field[8-14].

Thermoelectric transport properties have been revealed to be powerful tools in the study of intrinsic transport mechanisms in metals and semiconductors, providing complement to the inadequacy of conductivity measurements. Recently, a number of experiments have been carried out to investigate the thermoelectric properties of graphene. The expected change of sign of the thermopower is confirmed experimentally when the majority carriers change from electrons to holes in the absence of magnetic field[15-21]. When the magnetic field is lower than 10T and still strong enough to quantize the system, the Seebeck coefficient is found to has a sign anomaly in the lowest LL[15, 17-19]. Several theories have been put forward to explain these thermoelectric transport properties of graphene[2228]. However, many observed thermoelectric properties re-

\footnotetext{
${ }^{*}$ Corresponding author

Email address: duanwy@pku.edu.cn (Wenye Duan)
}

mained to be explained. Recent experimental and theoretical work have shown several different strategies to improve the figure of merit ZT [29-44]. For graphene under a perpendicular magnetic field, the confinement of electron motion in cyclotron orbital effectively reduce the dimensionality of the electronic states and an enhanced figure of merit is expected.

In this Letter, we present a numerical study of the thermoelectric and thermal transport properties of graphene with shortrange scattering impurities under strong magnetic field. Our result of the chemical potential dependence of many thermoelectric properties is in very good agreement with experimental observations. The single adjust parameter is the product of impurity potential strength and concentration. The only remaining anomaly is the Seebeck coefficient near zero chemical potential. Moreover, our study of the longitudinal and transverse thermal conductivities which are not measured in previous experiments may motivate further experimental investigations on the physical origin of the anomaly at the lowest LL. The large values of the thermoelectric figure of merit $Z T$ at $|n|=1$ LL suggest the potential application of graphene in thermoelectric devices.

\section{Formalism}

Based on the nearest-neighbor tight-binding model with the $\mathbf{k} \cdot \mathbf{p}$ method or with the effective-mass approximation[45-47], a graphene system with short-range impurity are described by

$$
H=v_{F}\left(\tau_{0} \otimes \sigma_{x} \pi_{x}+\tau_{z} \otimes \sigma_{y} \pi_{y}\right)+U(\mathbf{r}),
$$

on the basis $\Psi=\left(\psi_{A K}, \psi_{B K}, \psi_{A K^{\prime}},-\psi_{B K^{\prime}}\right)^{T}$, where $T$ stands for transpose, $\boldsymbol{\pi}=\mathbf{p}+e \mathbf{A}$ is the mechanical momentum (we use the SI units and electron charge is $-e(e>0)$ ), 


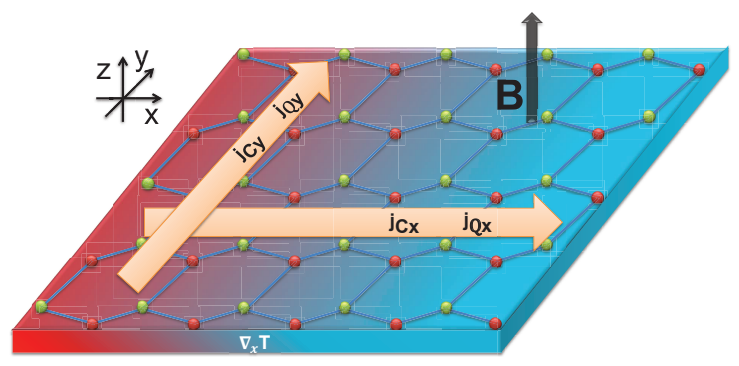

Figure 1: Schematic illustration of the system.

$v_{F}$ is the Fermi velocity, and $\mathbf{A}=(0, B x, 0)$ in the Laudau gauge, $U(\mathbf{r})=\sum_{i, \zeta} U_{i}^{\zeta}(\mathbf{r})$ with $U_{i}^{\zeta}(\mathbf{r})=(1 / 2)\left[\tau_{0} \pm\left(\tau_{x} \cos \phi_{i}^{\zeta}-\right.\right.$ $\left.\left.\tau_{y} \sin \phi_{i}^{\zeta}\right) / 2\right] \otimes\left(1 \pm \sigma_{z}\right) u_{i} \delta\left(\mathbf{r}-\mathbf{r}_{i}^{\zeta}\right)$ for a short-scatterer presented at site $\mathbf{r}_{i}^{\zeta}$ of sublattice $\zeta(\zeta=A$ corresponding (+) and $B$ corresponding (-)) and $\phi_{i}^{\zeta}=\left(\mathbf{K}^{\prime}-\mathbf{K}\right) \cdot \mathbf{r}_{i}^{\zeta}$ and $u_{i}$ is the strength of the impurity, $\tau_{0}$ and $\sigma_{0}$ is the identity matrix, $\tau_{a}$ and $\sigma_{a}(a=x, y, z)$ are the Pauli matrices for valley degree $\left(\mathbf{K}\right.$ and $\left.\mathbf{K}^{\prime}\right)$ and sublattice degree ( $A$ and $B$ ).

We obtain the Landau level basis by solving the Dirac equations analytically. The eigenstates of the Dirac equation are found as,

$$
\Psi_{n, k_{y}}^{\xi}(x, y)=C_{n} L_{y}^{-1 / 2} e^{i k_{y} y} \Phi_{n, k_{y}}^{\xi}(x),
$$

with $C_{n}=1$ for $n=0$ and $1 / \sqrt{2}$ for $n \neq 0$, specified in a set of quantum numbers $\gamma=$ $\left(\xi, n, k_{y}\right)$ with the eigenvalues $\epsilon_{n}=\operatorname{sgn}(n) \hbar \omega \sqrt{|n|}$, $\Phi_{n, k_{y}}^{+}(x)=\left(-i \operatorname{sgn}(n) \phi_{|n|-1, k_{y}}(x), \phi_{|n|, k_{y}}(x), 0,0\right)^{T}, \quad$ and $\Phi_{n, k_{y}}^{-}(x)=\left(0,0, \phi_{|n|, k_{y}}(x),-i \operatorname{sgn}(n) \phi_{|n|-1, k_{y}}(x)\right)^{T}, \operatorname{sgn}(x)$ is the sign function, and $\phi_{|n|, k_{y}}(x)=\exp \left[-\left(x / l_{B}+\right.\right.$ $\left.\left.k_{y} l_{B}\right)^{2} / 2\right]\left(2^{|n|}|n| ! \sqrt{\pi} l_{B}\right)^{-1 / 2} H_{|n|}\left(x / l_{B}+k_{y} l_{B}\right)$, where $l_{B}=\sqrt{\hbar / e B}$, $\hbar \omega=\sqrt{2} \hbar v_{F} / l_{B}$, and $\xi= \pm 1$ for the $\mathbf{K}(+)$ and $\mathbf{K}^{\prime}(-)$ valleys, respectively. $n$ is an integer number and $H_{n}(x)$ is the Hermite polynomial, $k_{y}$ is the wave vector along $y$ direction.

We assume that the average over random impurity configurations $\left\langle u_{i}^{2}\right\rangle=u^{2}$ and $n^{A}=n^{B}=n / 2$ and $n^{A}$ and $n^{B}$ being numbers of scatterers per unit area at site $A$ and site $B$ respectively. Considering the fact $\left|\mathbf{K}^{\prime}-\mathbf{K}\right| \gg k_{y}$ and after a straightforward calculations, in the self-consistent Born approximation (SCBA) the Green function $G(\epsilon)$ and self-energy $\Sigma(\epsilon)$ are proved to be diagonal with respect to the quantum numbers. Therefore, we have $G_{\gamma}(\epsilon)=\left[\epsilon-\epsilon_{\gamma}-\Sigma(\epsilon)\right]^{-1}$ and $\Sigma(\epsilon)=V(\hbar \omega)^{2} \sum_{\gamma} G_{\gamma}(\epsilon) / 2$, where $V=n u^{2} / 4 \pi\left(\hbar v_{F}\right)^{2}$ is the dimensionless parameter characterizing the scattering strength and concentration of impurities.

The system under consideration is schematically shown in Fig. 1. The charge current $\mathbf{j}_{\mathbf{C}}$ and heat current $\mathbf{j}_{\mathbf{Q}}$ induced by gradient of the electrochemical potential $\mathbf{E}^{*}=-\nabla(\phi+\mu / e)$ and temperature gradient are given as,

$$
\mathbf{j}_{\mathbf{C}}=\sigma \cdot \mathbf{E}^{*}-\alpha \cdot \nabla T,
$$

and

$$
\mathbf{j}_{\mathbf{Q}}=\boldsymbol{\alpha}^{\prime} \cdot \mathbf{E}^{*}-\lambda \cdot \nabla T,
$$

where $\sigma$ is the electrical conductivity tensor and $\alpha$ is the thermoelectric conductivity tensor and $\alpha^{\prime}=T \alpha$. The thermal properties are reflected by the following physical measurements: thermopower tensor $\mathbf{S}=\sigma^{-1} \cdot \boldsymbol{\alpha}$, thermal conductivity tensor $\boldsymbol{\kappa}=\boldsymbol{\lambda}-\boldsymbol{\alpha} \cdot \boldsymbol{\sigma}^{-1} \cdot \boldsymbol{\alpha}$, and the thermoelectric figure of merit $Z T=\sigma_{x x} S_{x x}^{2} T / \kappa_{x x}$. We calculate the transport coefficients by zero temperature conductivity $\sigma_{a b}(0, \epsilon)$ using the relation [48$50]$

$$
L_{a b}^{\beta \eta}(T, \mu)=\frac{-1}{T^{\eta}} \int d \epsilon f_{F}^{\prime}(\epsilon)\left(\frac{\epsilon-\mu}{-e}\right)^{\beta} \sigma_{a b}(0, \epsilon),
$$

where $\sigma_{a b}=L_{a b}^{00}, \alpha_{a b}=L_{a b}^{11}, \lambda_{a b}=L_{a b}^{21}(a, b=x, y)$, and $f_{F}(\epsilon)=\left[1+\exp (\epsilon-\mu) /\left(k_{B} T\right)\right]^{-1}$ is the Fermi-Dirac distribution. The electrical conductivity $\sigma_{a b}(0, \epsilon)=\sigma_{a b}^{I}(0, \epsilon)+\sigma_{a b}^{I I}(0, \epsilon)$, with $\sigma_{a b}^{I}(0, \epsilon)=$ $g_{s} e^{2} \hbar /\left(4 \pi L^{2}\right) \operatorname{Tr}\left\langle v^{a}\left(G^{+}-G^{-}\right) v^{b} G^{-}-v^{a} G^{+} v^{b}\left(G^{+}-G^{-}\right)\right\rangle$ and $\sigma_{a b}^{I I}(0, \epsilon)=g_{s} e^{2} /\left(4 i \pi L^{2}\right) \operatorname{Tr}\left\langle\left(r^{b} v^{a}-r^{a} v^{b}\right)\left(G^{+}-G^{-}\right)\right\rangle$, where $G^{ \pm}=G(\epsilon \pm i 0), g_{s}$ is the degeneracy of spin, $L^{2}$ is the area of the system, $\mathbf{r}$ and $\mathbf{v}$ are the position and velocity operators, respectively.

\section{Numerical results and discussion}

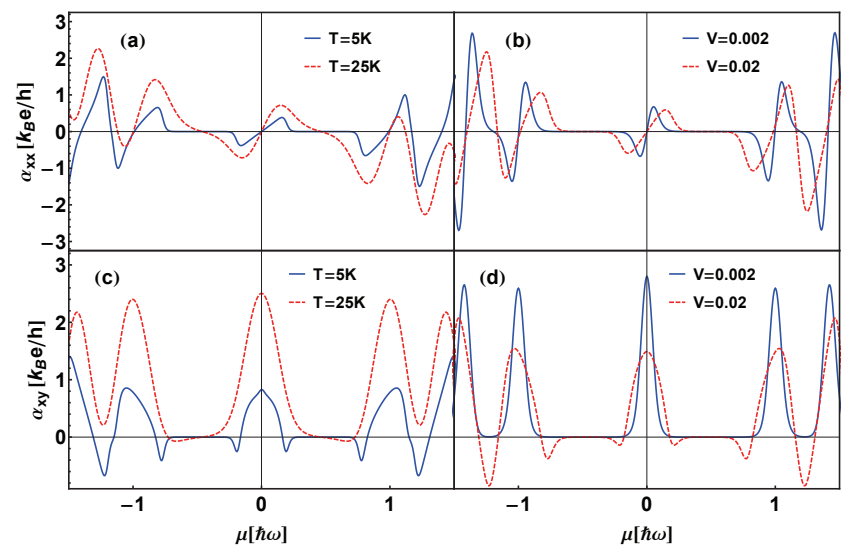

Figure 2: (a)The thermoelectric response function $\alpha_{x x}$, (c) $\alpha_{x y}$ as a function of $\mu$ with $V=0.02$ at different temperatures. (b) $\alpha_{x x}$, (d) $\alpha_{x y}$ as a function of $\mu$ with different $V$ at fixed temperature $T=10 \mathrm{~K}$.

The results for electrical and thermal transport coefficients with different impurity parameter as functions of the chemical potential at different temperatures are obtained numerically. We focus on the transport properties in the vicinity of the Dirac point in quantum limit, where the distance between LLs is greater than temperature and impurity broadening. These results are compared with experimental data and previous theoretical results. With only one adjustable parameter representing the impurity concentration and strength, our results are in good agreement with experimental results obtained previously. One exception is the Seebeck coefficients at zero chemical potential. This anomaly remains unresolved. Up to now, there is limited experimental result for the thermal conductivity under strong 
magnetic field. Our results may give a guide for further experiments on the thermoelectrics and thermal conductivities of graphene.

The thermoelectric conductivities $\alpha_{x x}$ and $\alpha_{x y}$ are shown in Fig. 2. $\alpha_{x x}$ is symmetric with respect to $\mu=0$ while $\alpha_{x y}$ is antisymmetric. Both $\alpha_{x x}$ and $\alpha_{x y}$ vanish when the chemical potential is located between the neighboring LLs. $\alpha_{x y}$ exhibits a single peak at the center of LLs. $\alpha_{x x}$ exhibits a drastic oscillation and alternates signs at the center of LLs. The behavior basically reproduces the features observed in experiments [18]. With increasing temperature $T$ or impurity parameter $V$, both the oscillation width in $\alpha_{x x}$ and the peak width in $\alpha_{x y}$ around each LL increase, which is consistent with the LL broadening.

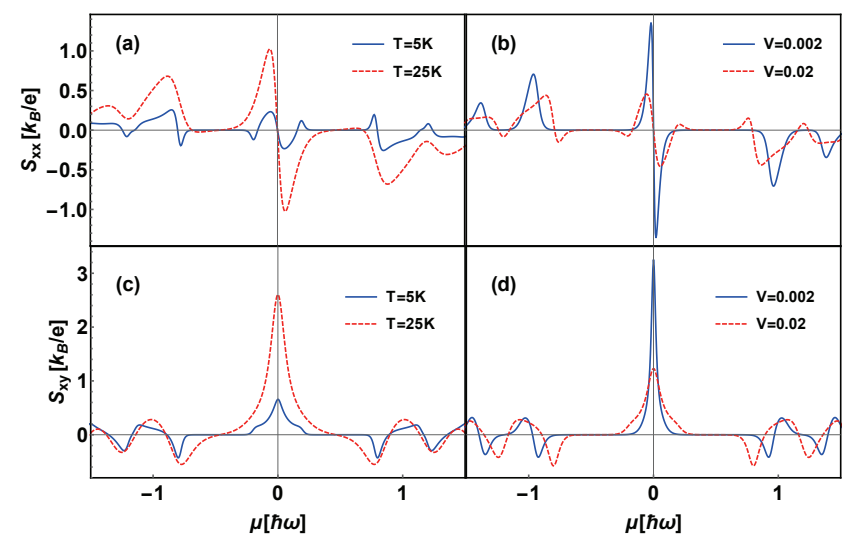

Figure 3: (a)The Seebeck coefficient $S_{x x}$, (c) Nernst coefficient $S_{x y}$ as a function of $\mu$ with $V=0.02$ at different temperatures. (b) $S_{x x}$, (d) $S_{x y}$ as a function of $\mu$ with different $V$ at fixed temperature $T=10 \mathrm{~K}$.

The Seebeck coefficient $S_{x x}$ and Nernst coefficient $S_{x y}$ are shown in Fig. 3. $S_{x x}$ is symmetric with respect to $\mu=0$ while $S_{x y}$ is antisymmetric. When $\mu$ is located between the neighboring LLs, both $S_{x x}$ and $S_{x y}$ vanish. When $\mu$ crosses each nonzero LL, $S_{x x}$ shows peaks when $\mu<0$ and dips when $\mu>0$, while $S_{x y}$ oscillates. At the $n=0 \mathrm{LL}, S_{x y}$ shows a peak while $S_{x x}$ shows an oscillation. In this oscillation, $S_{x x}$ shows a dip for $\mu>0$, but a peak for $\mu<0$. But the experimental results show that around the $n=0$ LL $S_{x x}$ has a dip for $\mu<0$ and a peak for $\mu>0[15,17,18]$. This discrepancy is related to the overestimation of longitudinal resistivity at the $n=0 \mathrm{LL}$, which also exists in other theoretical results obtained by different methods[24, 25]. $S_{x y}$ obtained with our model reproduces qualitatively the experimental results for the entire chemical potential range[15, 17, 18].

We proceed to analyze thermal conductivities as shown in Fig. 4. As a function of $\mu$, the thermal conductivity $\kappa_{x x}$ is symmetric with respect to $\mu=0$ while $\kappa_{x y}$ is antisymmetric. When $\mu$ crosses LLs, $\kappa_{x x}$ shows a double-peak structure at higher LLs $(|n|>0)$ at low temperature in near clear system as shown in Fig. 4(b). This phenomena was also predicted in other theoretical results[23, 27] where it was shown that the split $\kappa_{x x}$ produces antiphase oscillations with respect to the electric conductivities and leads to the violation of the Wiedemann-Franz law $L_{0}=\kappa_{x x} / \sigma_{x x} T$ where $L_{0}=\pi^{2} k_{B}^{2} /\left(3 e^{2}\right)$ as the comparison between the results from our numerical results and from

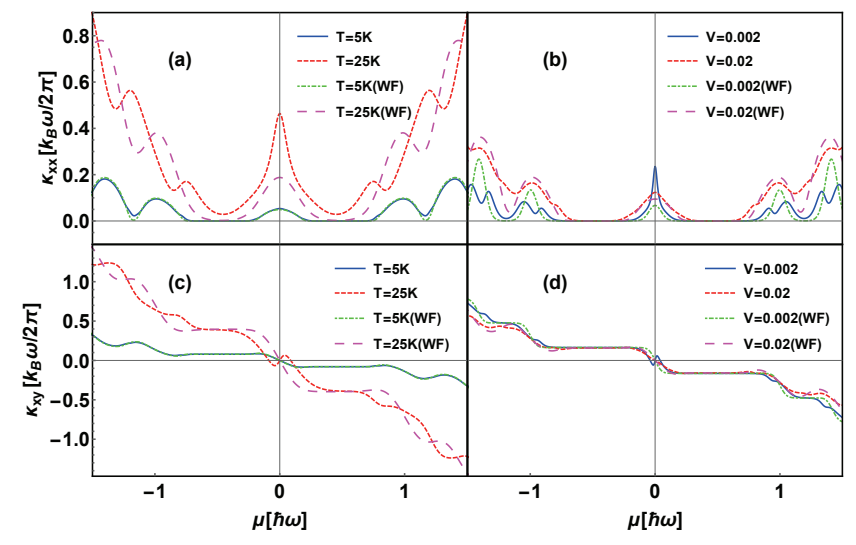

Figure 4: (a) The thermal conductivity $\kappa_{x x}$, and (c) thermal Hall conductivity $\kappa_{x y}$ a function of $\mu$ with $V=0.02$ at different temperatures. (b) $\kappa_{x x}$, and (d) $\kappa_{x y}$ as a function of $\mu$ with different $V$ at fixed temperature $T=10 \mathrm{~K}$. The results calculated from the Wiedemann-Franz law by $\sigma_{x x}$ are labeled by (WF) in the legends.

the Wiedmann-Franz law in Fig. 4(b). With increasing temperature, the double peak of $\kappa_{x x}$ smear to a single peak. However, we find that at low temperature with high impurity, the Wiedemann-Franz law is preserved as shown in Fig. 4(a) at $T=5 K$. When the Fermi energy is between two neighboring LLs, $\kappa_{x y}$ shows quantum plateaus. Besides, Fig. 4(d) shows that the quantized plateaus of $\kappa_{x y}$ is robust against impurities. It seems that the well known Wiedemann-Franz law is satisfied at the plateau regions of $\kappa_{x y}$. As shown in Fig. 4(c) and (d) at high temperature, $\kappa_{x y}$ changes sign as a function of $\mu$ at the lowest LL which is not shown in the case of zero magnetic field[51, 52] and similar phenomena are reported in bilayer graphene[53]. Though the thermal conductivity has been investigated experimentally in the absence of magnetic field $[51,52]$, there still lack experimental results of the longitudinal and transverse thermal conductivity under magnetic fields. Our results of the longitudinal resistivity and the chemical potential dependence of the Seebeck coefficients at the lowest LL are in good qualitative agreement with experimental results of high mobility graphene samples [16]. The anomalous oscillation behavior at the lowest LL and the quantum plateaus of the transverse thermal conductivity and double peaks of longitudinal thermal conductivity at the higher LL are predicted to be observed in experimental measurements in clear samples with high mobility.

Finally, we discuss the thermoelectric figure of merit $Z T$. Even though the Landau quantization are not readily emergent at temperatures in which the domestic thermoelectric refrigeration is used, the properties we obtained can be useful in cooling and power generation application at low temperatures. We show the thermoelectric figure of merit $Z T$ in Fig. 5. The thermoelectric figure of merit $Z T$ is symmetric with respect to $\mu=0$, show peaks at high LLs and double peak at the lowest LL. Because of the double-peak structure in the longitudinal thermal conductivity and the violation of the WiedemannFranz law, the thermoelectric figure of merit $Z T$ show an obvious strong peak at the first LL with the order $Z T \backsim 1$ which 


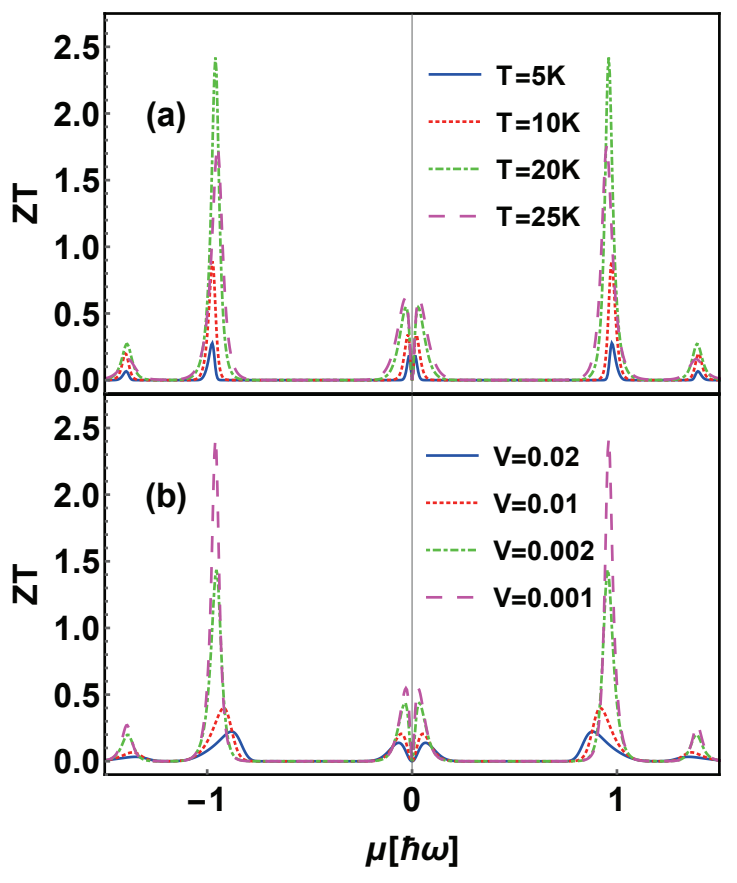

Figure 5: The thermoelectric figure of merit $Z T$ as a function of $\mu$ (a) for different temperature with fixed $V=0.001$ and (b) for different $V$ with fixed temperature $T=20 K$.

is dramatically improved compared with $Z T \backsim 0.01$ for monolayer graphene ribbons[29]. The thermoelectric figure of merit $Z T$ reaches 2.4 for $V=0.001$ at $|n|=1$ Landau level with $T=20 K$. This is regarded as superior thermoelectric property and can significantly expand the thermoelectric application of graphene[54, 55]. The results suggest that an external strong magnetic field could be used to effectively enhance the $Z T$.

\section{Conclusion}

In summary, we numerically studied the thermal and thermoelectric properties of graphene under a quantising magnetic field and in the presence of short range scatterers. The thermoelectric conductivity tensor, Seebeck and Nernst coefficients, thermal conductivities, and the thermoelectric figure of merit are obtained. Our results agree well with the experimental data, except for the Seebeck coefficient on the zeroth LL. The anomaly in Seebeck coefficient has been also reported in precious theoretical results by different methods and may be related to the overestimation of the longitudinal resistivity. It implies that the short-range impurity scattering cannot account for the anomalous Seebeck coefficient at the zeroth LL. Our numerical results of the longitudinal and transverse thermal conductivity, especially the double peak structure of $\kappa_{x x}$ at high LLs and the anomalous oscillation at the lowest LL and quantum plateaus of $\kappa_{x y}$ should stimulate further experimental study on the thermal and thermoelectric properties of high mobility graphene samples. This study is useful to further investigations in the physical origin of the anomalous transport properties of graphene at the lowest LL as our results clearly indicate that the anomaly is not accounted for by the short-range scattering. The large values of the thermoelectric figure of merit $Z T$ at $|n|=1$ Landau level suggest that the potential application of graphene in thermoelectric devices is huge.

\section{Acknowledgments}

The authors are thankful for the support of NSFC (11274013,11774006), NBRP of China (2012CB921300), and the Australian Research Council Grant (DP160101474).

\section{References}

[1] K.S. Novoselov et al., Science 306 (2004) 666

[2] K. S. Novoselov et al., Nature (London) 438 (2005) 197.

[3] M. I. Katsnelson, K. S. Novoselov, and A. K. Geim, Nat. Phys. (London) 2 (2006) 620.

[4] A. H. Castro Neto, F. Guinea, N. M. R. Peres, K. S. Novoselov, and A. K. Geim, Rev. Mod. Phys. 81 (2009) 109.

[5] S. D. Sarma, S. Adam, E. H. Hwang, and E. Rossi, Rev. Mod. Phys. 83 (2011) 407.

[6] Y. Zhang, Y.W. Tan, H.L. Stormer, and P. Kim, Nature (London) 438 (2005) 201.

[7] L. Zhang, Y. Zhang, M. Khodas, T. Valla, and I. A. Zaliznyak, Phys. Rev. Lett. 105 (2010) 046804.

[8] Y. Zhang et al., Phys. Rev. Lett. 96 (2006) 136806.

[9] Z. Zhang, Y. Zhang, H. L. Stormer, and P. Kim, Phys. Rev. Lett. 99 (2007) 106802 .

[10] D. A. Abanin, K. S. Novoselov, U. Zeilter, P. A. Lee, A. K. Geim, and L. S. Levitov, Phys. Rev. Lett. 98 (2007) 196806.

[11] J. G. Checkelsky, Lu Li, and N. P. Ong, Phys. Rev. Lett. 100 (2008) 206801; Phys. Rev. B 79 (2009) 115434.

[12] A. J. M. Giesbers et al., Phys. Rev. B. 80 (2009) 201403(R).

[13] K. I. Bolotin, F. Ghahari, M. D. Shulman, H. L. Stormer, and P. Kim, Nature(London) 462 (2009) 196.

[14] X. Du, I. Skachko, F. Duerr, A. Luican, and E. Y. Andrei, Nature(London) 462 (2009) 192.

[15] P. Wei, W. Bao, Y. Pu, C. N. Lau, and J. Shi, Phys. Rev. Lett. 102 (2009) 166808.

[16] X. Liu, D. Wang, P. Wei, L. Zhu, and J. Shi, Phys. Rev. B 86 (2012) 155414.

[17] Y. M. Zuev, W. Chang, and P. Kim, Phys. Rev. Lett. 102 (2009) 096807.

[18] J. G. Checkelsky and N. P. Ong, Phys. Rev. B 80 (2009) 081413(R).

[19] Y. Nam, J. Sun, N. Lindvall, S. J. Yang, C. R. Park, Y. W. Park, and A. Yurgens, Appl. Phys. Lett. 104 (2014) 021902.

[20] D. Wang, and J. Shi, Phys. Rev. B 83 (2011) 113403.

[21] F. Ghahari, H.- Y. Xie, T. Taniguchi, K. Watanabe, M. S. Foster, and P. Kim, Phys. Rev. Lett. 116 (2016) 136802.

[22] V. P. Gusynin and S. G. Sharapov, Phys. Rev. B 71 (2005) 125124.

[23] B. Dóra and P. Thalmeier, Phys. Rev. B 76 (2007) 035402.

[24] L. Zhu, R. Ma, L. Sheng, M. Liu, and D.-N. Sheng, Phys. Rev. Lett. 104 (2010) 076804

[25] S. -P. Chao and V. Aji, Phys. Rev. B 84 (2011) 155430.

[26] V. Ugarte, V. Aji, and C. M. Varma, Phys. Rev. B 84 (2011) 165429.

[27] R Nasir and K Sabeeh J. Phys: Condens. Matt 231 (2011) 37530.

[28] I. Proskurin and M. Ogata, J. Phys. Soc. Jpn. 82 (2013) 063712.

[29] H. Sadeghi, S. Sangtarash, and C. J. L ambert, Beilstein J. Nanotechnol. 6 (2015) 1176.

[30] H. Zheng, H. J. Liu, X. J. Tan, H. Y. Lv, L. Pan, J. Shi, and X. F. Tang, Appl. Phys. Lett. 100 (2012) 093104.

[31] D. Olaya, M. H.- Morales, D. Gómez, O. A. C.-Uribe, Z.-Y. Juang, and Y. Hernández, 2D Mater. 5 (2018) 011004

[32] S.Yadav, S. Chaudhary, and D. K. Pandya, Ceramics International 44 (2018) 10628

[33] X. Cheng and G.-Y. Sun, Physica E 87 (2017) 84.

[34] M. C. Nguyen, V. H. Nguyen, H.-V. Nguyen, J. S.-Martin, and P. Dollfus, Physica E 73 (2015) 207.

[35] S.I.Vishkayi and H. R. Soleimani, Physica E 74 (2015) 363.

[36] U. Patil and B. Muralidharan, Physica E 85 (2017) 27. 
[37] R. D’Souza and S. Mukherjee, Physica E 81 (2016) 96.

[38] S. T. Rodriguez, I. Grosu, M. Crisan, and I. Tifrea, Physica E 96 (2018)

[39] S. M.-Valdovinos, J. M.-Rivera, N. E. M.-Cabrera, and I. R.-Vargas, Physica E 101 (2018) 188.

[40] V. -T. Tran, J. S.-Martin, P. Dollfus, and S. Volz, Scientific Report 7 (2017) 2313.

[41] Y. Anno, Y. Imakita, K. Takei, S.Akita, and T. Arie, 2D Mater. 4 (2017) 025019 .

[42] K.Zberecki, R. Swikowicz, M. Wierzbicki, and J. Barnas, Phys. Chem Chem. Phys. 18 (2016) 18246.

[43] D.V. Kolesnikov, D.A. Lobanov, and V.A. Osipov, Solid State Common. 248 (2016) 83.

[44] J. Y. Kim and J. C. Grossman, Nano Lett. 15 (2015) 2830.

[45] T. Ando, J. Phys. Soc. Jpn. 74 (2005) 777.

[46] N. H. Shon and T. Ando, J. Phys. Soc. Jpn. 67 (1998) 2421

[47] Y. Zheng and T. Ando, Phys. Rev. B 65 (2002) 245420.

[48] L. Smreka and P. Strěda, J. Phys.: Solide State Phys. C 10 (1977) 2153.

[49] H. Oji and P. Strěda, Phys. Rev. B 31 (1985) 7291.

[50] M. Jonson and S.M.Girvin, Phys. Rev. B 29 (1984) 1939.

[51] S. Yiğen and A.R. Champagne, Nano Lett. 14 (2014) 289.

[52] J. Crossno et al., Science 351 (2016) 1058.

[53] R. Ma, L. Zhu, L.Sheng, M. Liu, and D. N. Sheng, Phys. Rev. B 84 (2011) 075420

[54] C. B. Vining, Nat. Mater. 8 (2009) 83.

[55] T. M. Tritt, Annu. Rev. Mater. Res. 41 (2011) 433. 